\title{
Cohomological dimension of complexes
}

\author{
Mohammad T. Dibaei ${ }^{a b}$ and Siamak Yassemi ${ }^{c a}$ \\ (a) Institute for Studies in Theoretical Physics and Mathematics \\ (b) Department of Mathematics, Teacher Training University \\ (c) Department of Mathematics, University of Tehran.
}

\begin{abstract}
In the derived category of the category of modules over a commutative Noetherian ring $R$, we define, for an ideal $\mathfrak{a}$ of $R$, two different types of cohomological dimensions of a complex $X$ in a certain subcategory of the derived category, namely $\operatorname{cd}(\mathfrak{a}, X)=\sup \left\{\operatorname{cd}\left(\mathfrak{a}, \mathrm{H}_{\ell}(X)\right)-\ell \mid \ell \in \mathbb{Z}\right\}$ and $-\inf \mathbf{R} \Gamma_{\mathfrak{a}}(X)$, where $\operatorname{cd}(\mathfrak{a}, M)=$ $\sup \left\{\ell \in \mathbb{Z} \mid \mathrm{H}_{\mathfrak{a}}^{\ell}(M) \neq 0\right\}$ for an $R$-module $M$. In this paper, it is shown, among other things, that, for any complex $X$ bounded to the left, $-\inf \mathbf{R} \Gamma_{\mathfrak{a}}(X) \leq \operatorname{cd}(\mathfrak{a}, X)$ and equality holds if indeed $\mathrm{H}(X)$ is finitely generated.
\end{abstract}

\section{Introduction}

Let $R$ be a commutative Noetherian ring of finite dimension $d$ and $\mathfrak{a}$ be an ideal of $R$. For an $R$-module $M, \Gamma_{\mathfrak{a}}(M)$ is defined to be the submodule of $M$ consisting of all elements of $M$ which are vanished by some power of $\mathfrak{a}$. It has been an interesting question to know when $\mathrm{H}_{\mathfrak{a}}^{i}(M)$, the $\mathrm{i}$-th right derived functor of $\Gamma_{\mathfrak{a}}(-)$ applied on $M$, is zero (see $[\mathbf{H u}])$. The cohomological dimension of $M$ with respect to $\mathfrak{a}$ is defined as

$$
\operatorname{cd}(\mathfrak{a}, M)=\sup \left\{i \in \mathbb{Z} \mid \mathrm{H}_{\mathfrak{a}}^{i}(M) \neq 0\right\} .
$$

2000 Mathematics subject classification. 13D45.

Key words and phrases. Local cohomology, cohomological dimension, complexes of modules. 
In [G] Grothendieck has shown that $\mathrm{cd}(\mathfrak{a}, M)$ has a lower bound and an upper bound $\operatorname{depth} M$ and $\operatorname{dim} M$ respectively. The cohomological dimension has been studied by several authors. In $[\mathbf{F a}]$ Falting and in $[\mathbf{H L}]$ Huneke-Lyubeznik have found several upper bounds for cohomological dimension. In [DNT], some lower bounds have been obtained.

In section 1 , we study the cohomological dimension of a module in more details. We show that for an $R$-module $M, \operatorname{cd}(\mathfrak{a}, M) \leq \operatorname{cd}(\mathfrak{a}, R / \mathfrak{p})$ for some $\mathfrak{p} \in \operatorname{Supp}_{R}(M)$ and equality holds if $M$ is finite (that is finitely generated) and in this case $\mathfrak{p}$ may be taken from the set of minimal elements of $\operatorname{Supp}_{R}(M)$ (Theorem 1.3).

In the derived category $\mathcal{D}(R)$, for a complex $X$, the $\mathfrak{a}$-depth of $X$ is defined by the following formula, cf. [I; Section 2]:

$$
\operatorname{depth}(\mathfrak{a}, X)=\inf \left\{\ell \in \mathbb{Z} \mid \mathrm{H}_{-\ell}\left(\mathbf{R H o m}{ }_{R}(R / \mathfrak{a}, X)\right) \neq 0\right\} .
$$

In [FI; Theorem 2.1], Foxby and Iyengar have shown that, for any complex $X$,

$$
\operatorname{depth}(\mathfrak{a}, X)=-\sup \mathbf{R} \Gamma_{\mathfrak{a}}(X) .
$$

In this paper we are specially interested in $\inf \mathbf{R} \Gamma_{\mathfrak{a}}(X)$. It is clear that for an $R$-module $M,-\inf \mathbf{R} \Gamma_{\mathfrak{a}}(M)=\mathrm{cd}(\mathfrak{a}, M)$ and so by Grothendieck's result, we have $-\inf \mathbf{R} \Gamma_{\mathfrak{a}}(M) \leq$ $\operatorname{dim}(M)$. What is cited is our motivation to study the invariant $-\inf \mathbf{R} \Gamma_{\mathfrak{a}}(X)$. On the other hand, for a complex $X$ bounded to the left, in consistent with the definition of the dimension of $X$ as $\operatorname{dim} X=\sup \left\{\operatorname{dim}_{R} \mathrm{H}_{\ell}(X)-\ell \mid \ell \in \mathbb{Z}\right\}$, we may define cohomological dimension of $X$ as $\operatorname{cd}(\mathfrak{a}, X)=\sup \left\{\operatorname{cd}\left(\mathfrak{a}, \mathrm{H}_{\ell}(X)\right)-\ell \mid \ell \in \mathbb{Z}\right\}$. Our purpose is to clarify the relationship between $\inf \mathbf{R} \Gamma_{\mathfrak{a}}(X)$ and $\operatorname{cd}(\mathfrak{a}, X)$.

We first study $\operatorname{cd}(\mathfrak{a}, X)$ in section 2 . As $\operatorname{cd}(\mathfrak{a}, M)$ is well behaved when $M$ is a finite module, most results of this section are in the case $X$ has finite homology modules, for example: If $X, Y$ are complexes bounded to the left with finite homology modules then

$$
\operatorname{cd}\left(\mathfrak{a}, X \otimes_{R}^{\mathbf{L}} Y\right)=\sup \left\{\operatorname{cd}\left(\mathfrak{a}, \mathrm{H}_{t}(X) \otimes_{R} \mathrm{H}_{\ell}(Y)\right)-\ell-t \mid t, \ell \in \mathbb{Z}\right\} \quad \text { (see Theorem 2.9), }
$$

and we have

$$
\operatorname{cd}\left(\mathfrak{a}, X \otimes_{R}^{\mathbf{L}} Y\right) \leq \operatorname{cd}(\mathfrak{a}, X)-\inf Y \quad(\text { see Theorem 2.10). }
$$


Moreover, there are some results which $X$ does not need to have finite homology modules, for example: If $X$ is a bounded to the left complex, then

$$
\operatorname{cd}(\mathfrak{a}, X) \leq \operatorname{cd}(\mathfrak{a}, R)-\inf X \quad \text { (see Proposition 2.5). }
$$

In section 3, we compare $\operatorname{cd}(\mathfrak{a}, X)$ with $-\inf \mathbf{R} \Gamma_{\mathfrak{a}}(X)$. More precisely, we show that for any bounded to the left complex $X$ the invariant $-\inf \mathbf{R} \Gamma_{\mathfrak{a}}(X)$ has a lower and an upper bounds depth $(\mathfrak{a}, X)$ and $c d(\mathfrak{a}, X)$ respectively; and it takes its upper bound when indeed the homology modules of $X$ are finite (see Theorem 3.2 and Theorem 3.3).

\section{Cohomological dimension of a module}

In this section, we have a brief look at the cohomological dimension of a module to find some extra properties (see Theorem 1.2) and to give an extension of [DNT; Theorem 2.2] (see Theorem 1.5).

First recall the well-known fact about $\mathrm{cd}(\mathfrak{a}, R)$ which states that

(1.0.1) For any $R$-module $M, \operatorname{cd}(\mathfrak{a}, M) \leq \mathrm{cd}(\mathfrak{a}, R)$.

The following result will be helpful to remove the finiteness condition on the modules.

Theorem 1.1. If $M$ is an $R$-module, then

$$
\operatorname{cd}(\mathfrak{a}, M) \leq \sup \{\operatorname{cd}(\mathfrak{a}, N) \mid N \text { is a finite submodule of } M\} .
$$

Proof. It is well-known that $M$ is equal to the direct limit of its finite submodules. Now the assertion follows from the fact that the local cohomology functor commutes with the direct limit.

Lemma 1.2. If $M$ is an $R$-module and $\mathfrak{b}$ is an ideal with $\mathfrak{b} \subseteq \operatorname{Ann}_{R}(M)$, then

$$
\operatorname{cd}(\mathfrak{a}, M) \leq \operatorname{cd}(\mathfrak{a}, R / \mathfrak{b}) .
$$

Proof. As $M$ is an $R / \mathfrak{b}$-module, we have

$$
\begin{aligned}
\operatorname{cd}(\mathfrak{a}, M) & =\operatorname{cd}(\mathfrak{a}(R / \mathfrak{b}), M) \\
& \leq \operatorname{cd}(\mathfrak{a}(R / \mathfrak{b}), R / \mathfrak{b}) \\
& =\operatorname{cd}(\mathfrak{a}, R / \mathfrak{b}) .
\end{aligned}
$$


The equalities hold by independence theorem on local cohomology and the inequality holds by (1.0.1).

Theorem 1.3. If $M$ is an $R$-module with finite cohomological dimension with respect to $\mathfrak{a}$, then $\operatorname{cd}(\mathfrak{a}, M) \leq \operatorname{cd}(\mathfrak{a}, R / \mathfrak{p})$ for some $\mathfrak{p} \in \operatorname{Supp}_{R}(M)$. Moreover, if $M$ is finite, the equality holds and $\mathfrak{p}$ can be taken from the set, $\operatorname{MinSupp}_{R}(M)$, of minimal elements of $\operatorname{Supp}_{R}(M)$.

Proof. By Theorem 1.1, there exists a finite submodule $K$ of $M$ such that $\operatorname{cd}(\mathfrak{a}, M) \leq$ $\operatorname{cd}(\mathfrak{a}, K)$, so it is enough to show the assertion for $K$. Assume the contrary. There is a chain $0=K_{0} \subset K_{1} \subset \cdots \subset K_{n}=K$ of submodules of $K$ such that, for each $i$, $K_{i} / K_{i-1} \cong R / \mathfrak{p}_{i}$, where $\mathfrak{p}_{i} \in \operatorname{Supp}_{R}(K)$. Set $t=\operatorname{cd}(\mathfrak{a}, K)$, so we have $\mathrm{H}_{\mathfrak{a}}^{t}\left(R / \mathfrak{p}_{i}\right)=0$ for $1 \leq i \leq n$. Thus from the exact sequences $\mathrm{H}_{\mathfrak{a}}^{t}\left(K_{i-1}\right) \rightarrow \mathrm{H}_{\mathfrak{a}}^{t}\left(K_{i}\right) \rightarrow 0, i=1,2, \ldots, n$, we eventually get $\mathrm{cd}\left(\mathfrak{a}, K_{1}\right) \geq t$ which is a contradiction.

Let $M$ be finite and so $\operatorname{cd}(\mathfrak{a}, M) \leq \operatorname{cd}(\mathfrak{a}, R / \mathfrak{p})$ for some $\mathfrak{p} \in \operatorname{Supp}_{R}(M)$. On the other hand $\operatorname{cd}(\mathfrak{a}, R / \mathfrak{p}) \leq \operatorname{cd}(\mathfrak{a}, M)$, c.f. [DNT; Theorem 2.2]. Hence the equality holds.

If $\mathfrak{p}$ does not belong to the set $\operatorname{MinSupp}_{R}(M)$ then there exists $\mathfrak{q} \in \operatorname{MinSupp}_{R}(M)$ with $\mathfrak{q} \subset \mathfrak{p}$. Now by using Lemma 1.2 , we have that $\operatorname{cd}(\mathfrak{a}, R / \mathfrak{p}) \leq \operatorname{cd}(\mathfrak{a}, R / \mathfrak{q})$, that is $\operatorname{cd}(\mathfrak{a}, R / \mathfrak{q})=\operatorname{cd}(\mathfrak{a}, M)$, and the claim follows.

The following result is a generalization of [DNT; Theorem 2.2].

Theorem 1.4. Let $N$ and $M$ be $R$-modules and $M$ finite. If $\operatorname{Supp}_{R}(N) \subseteq \operatorname{Supp}_{R}(M)$, then $\operatorname{cd}(\mathfrak{a}, N) \leq \operatorname{cd}(\mathfrak{a}, M)$.

Proof. By Theorem 1.1, $\operatorname{cd}(\mathfrak{a}, N) \leq \operatorname{cd}(\mathfrak{a}, K)$ for some finite submodule $K$ of $N$. Since $\operatorname{Supp}_{R}(K) \subseteq \operatorname{Supp}_{R}(M)$, we have by [DNT; Theorem 2.2], that $\operatorname{cd}(\mathfrak{a}, K) \leq \operatorname{cd}(\mathfrak{a}, M)$.

Corollary 1.5. Let $\varphi: R \rightarrow S$ be a ring homomorphism and let $M$ be a finite $R$-module. Then

$$
\operatorname{cd}_{S}\left(\mathfrak{a} S, S \otimes_{R} M\right) \leq \operatorname{cd}_{R}(\mathfrak{a}, M) .
$$

If $S$ is faithfully flat then the equality holds. 
Proof. By independence theorem $\mathrm{H}_{\mathfrak{a}}^{i}\left(S \otimes_{R} M\right) \cong \mathrm{H}_{\mathfrak{a} S}^{i}\left(S \otimes_{R} M\right)$ as $R$-modules. As $\operatorname{Supp}_{R}\left(S \otimes_{R} M\right) \subseteq \operatorname{Supp}_{R}(M)$, we have, by Theorem 1.1, $\operatorname{cd}_{S}\left(\mathfrak{a} S, S \otimes_{R} M\right) \leq \operatorname{cd}_{R}(\mathfrak{a}, M)$. The final claim is clear.

The following example shows that the finiteness condition on $M$ is not redundant in Theorem 1.4 and second part of Theorem 1.3.

Example 1.6. Choose a ring $R$, a prime ideal $\mathfrak{p}$ and an ideal $\mathfrak{a}$ such that $\mathfrak{a} \nsubseteq \mathfrak{p}$. We have $\operatorname{Supp}_{R}(R / \mathfrak{p}) \subseteq \operatorname{Supp}_{R}(\mathrm{E}(R / \mathfrak{p}))$, where $\mathrm{E}(R / \mathfrak{p})$ is the injective envelope of $R / \mathfrak{p}$ as $R$-module. We observe that $\operatorname{cd}(\mathfrak{a}, \mathrm{E}(R / \mathfrak{p}))=0$ but $0<\operatorname{cd}(\mathfrak{a}, R / \mathfrak{p})$.

\section{Cohomological dimension of a complex}

An $R$-complex $X$ is a sequence of $R$-modules $X_{\ell}$ and $R$-linear maps $\partial_{\ell}^{X}, \ell \in \mathbb{Z}$,

$$
X=\cdots \longrightarrow X_{\ell+1} \stackrel{\partial_{\ell+1}^{X}}{\longrightarrow} X_{\ell} \stackrel{\partial_{\ell}^{X}}{\longrightarrow} X_{\ell-1} \longrightarrow \cdots .
$$

The module $X_{\ell}$ is called the module in degree $\ell$, and the map $\partial_{\ell}^{X}: X_{\ell} \longrightarrow X_{\ell-1}$ is the $\ell$-th differential, and $\partial_{\ell}^{X} \partial_{\ell+1}^{X}=0$ for all $\ell \in \mathbb{Z}$. An $R$-module $M$ is thought of as the complex $M=0 \longrightarrow M \longrightarrow 0$,with $M$ in degree zero.

The supremum and infimum of $X$ are defined by

$$
\begin{aligned}
\sup X & =\sup \left\{\ell \in \mathbb{Z} \mid \mathrm{H}_{\ell}(X) \neq 0\right\} \\
\inf X & =\inf \left\{\ell \in \mathbb{Z} \mid \mathrm{H}_{\ell}(X) \neq 0\right\}
\end{aligned}
$$

Denote $\sup X=-\infty$ and $\inf X=\infty$ if $\mathrm{H}_{\ell}(X)=0$ for all $\ell$.

A morphism $\alpha: X \longrightarrow Y$ is said to be a quasi-isomorphism if the induced morphism $\mathrm{H}(\alpha): \mathrm{H}(X) \longrightarrow \mathrm{H}(Y)$ is an isomorphism.

The derived category $\mathcal{D}(R)$ of the category of $R$-complexes is the category of $R-$ complexes localized at the class of all quasi-isomorphisms. The full subcategories $\mathcal{D}_{+}(R)$, $\mathcal{D}_{-}(R), \mathcal{D}_{b}(R)$, and $\mathcal{D}_{0}(R)$ consist of complexes $X$ with $\mathrm{H}_{\ell}(X)=0$ for, respectively, $\ell \ll 0, \ell \gg 0,|\ell| \gg 0$, and $\ell \neq 0$. By $\mathcal{D}^{f}(R)$ we mean the full subcategory of $\mathcal{D}(R)$ consisting of complexes $X$ with $\mathrm{H}_{\ell}(X)$ a finite $R$-module for all $\ell$. 
The left derived functor of the tensor product functor of $R$-complexes is denoted by $-\otimes_{R}^{\mathbf{L}}-$, and $\mathbf{R} \operatorname{Hom}_{R}(-,-)$ denotes the right derived functor of the homomorphism functor of complexes. We need the next two inequalities for $X, Y \in \mathcal{D}_{+}(R)$ and $Z \in \mathcal{D}(R)$.

$$
\begin{aligned}
& \inf \left(X \otimes_{R}^{\mathbf{L}} Y\right) \geq \inf X+\inf Y \text { and } \\
& \sup \left(\mathbf{R H o m} \operatorname{Hom}_{R}(X, Z)\right) \leq \sup Z-\inf X .
\end{aligned}
$$

For a complex $X$, the dimension of $X$ is defined by

(2.0.3) $\operatorname{dim}_{R} X=\sup \left\{\operatorname{dim} R / \mathfrak{p}-\inf X_{\mathfrak{p}} \mid \mathfrak{p} \in \operatorname{Spec} R\right\}$.

It is shown, in $[$ Fo1; 16.9], that

$$
\operatorname{dim} X=\sup \left\{\operatorname{dim}_{R} \mathrm{H}_{\ell}(X)-\ell \mid \ell \in \mathbb{Z}\right\} .
$$

Therefore it is natural to give the following definition:

Definition 2.1. For a complex $X \in \mathcal{D}(R)$, the $\mathfrak{a}$-cohomological dimension of $X$ is defined by

$$
\operatorname{cd}(\mathfrak{a}, X)=\sup \left\{\operatorname{cd}\left(\mathfrak{a}, \mathrm{H}_{\ell}(X)\right)-\ell \mid \ell \in \mathbb{Z}\right\} .
$$

For an $R$-module $M$, this notion agrees with the classical one. Note that $\operatorname{cd}(\mathfrak{a}, X)=-\infty$ if and only if $X$ is homologically trivial. If $\inf X=-\infty$ then $\operatorname{cd}(\mathfrak{a}, X)=\infty$.

To find some extra information about $\mathrm{cd}(\mathfrak{a}, X)$, we also review the notion of the height of an ideal $\mathfrak{a}$. If $M$ is an $R$-module, the $M$-height of $\mathfrak{a}$, denoted by ht $(\mathfrak{a}, M)$, is defined to be the supremum length of chains $\mathfrak{p}_{0} \subset \mathfrak{p}_{1} \subset \cdots \subset \mathfrak{p}_{n}$ of elements of $\operatorname{Supp}_{R} M$ with $\mathfrak{p}_{n}$ is minimal over $\mathfrak{a}$. Thus, when $M$ is finite, we may write ht $(\mathfrak{a}, M)=\mathrm{ht}(\mathfrak{a}, R / \mathfrak{p})$ for some $\mathfrak{p} \in \operatorname{Supp}_{R} M$, and that

$$
\text { ht }(\mathfrak{a}, M)=\sup \left\{\text { ht }(\mathfrak{a}, R / \mathfrak{p}) \mid \mathfrak{p} \in \operatorname{Supp}_{R} M\right\} \text {. }
$$

If $M$ is zero module then ht $(\mathfrak{a}, M)=-\infty$ by convention.

Definition 2.2. For $X \in \mathcal{D}_{+}(R)$, the $X$-height of $\mathfrak{a}$ is defined by

$$
\text { ht }(\mathfrak{a}, X)=\sup \left\{\operatorname{ht}\left(\mathfrak{a}, \mathrm{H}_{\ell}(X)\right)-\ell \mid \ell \in \mathbb{Z}\right\} .
$$

Now we have the following result. 
Lemma 2.3. If $X \in \mathcal{D}_{+}^{f}(R)$, then

$$
\text { ht }(\mathfrak{a}, X)=\sup \left\{\operatorname{ht}(\mathfrak{a}, R / \mathfrak{p})-\inf X_{\mathfrak{p}} \mid \mathfrak{p} \in \operatorname{Spec} R\right\} \text {. }
$$

Proof. Consider $\ell \in \mathbb{Z}$ such that $\mathrm{H}_{\ell}(X) \neq 0$. There exists $\mathfrak{p} \in \operatorname{Supp}_{R} \mathrm{H}_{\ell}(X)$ with ht $(\mathfrak{a}, R / \mathfrak{p})=\operatorname{ht}\left(\mathfrak{a}, \mathrm{H}_{\ell}(X)\right)$. Thus ht $\left(\mathfrak{a}, \mathrm{H}_{\ell}(X)\right)-\ell \leq \operatorname{ht}(\mathfrak{a}, R / \mathfrak{p})-\inf X_{\mathfrak{p}}$ which gives a one side inequality.

For the other side, assume $\mathfrak{p} \in \operatorname{Supp}_{R} X$ and that $\ell=\inf X_{\mathfrak{p}}$. Thus ht $(\mathfrak{a}, R / \mathfrak{p}) \leq$ ht $\left(\mathfrak{a}, \mathrm{H}_{\ell}(X)\right)$. Now the assertion holds.

The following proposition compares the invariants $\operatorname{cd}(\mathfrak{a}, X), \operatorname{cd}(\mathfrak{a}, R), \operatorname{dim}_{R} X$, and ht $(\mathfrak{a}, X)$.

Proposition 2.4. For $X \in \mathcal{D}_{+}(R)$ the following hold.

(a) $\operatorname{cd}(\mathfrak{a}, X) \leq \operatorname{cd}(\mathfrak{a}, R)-\inf X$;

(b) $\operatorname{cd}(\mathfrak{a}, X) \leq \operatorname{dim}_{R} X$.

In addition, if $X \in \mathcal{D}_{+}^{f}(R)$ then

(c) ht $(\mathfrak{a}, X) \leq \operatorname{cd}(\mathfrak{a}, X)$.

Proof. (a) and (b) are consequences of (1.0.1) and the Grothendieck vanishing theorem on local cohomology. Part (c) follows from the well-known fact that ht $(\mathfrak{a}, N) \leq \operatorname{cd}(\mathfrak{a}, N)$ for any finite $R$-module $N$.

Proposition 2.5. If $X \in \mathcal{D}_{+}(R)$, then

$$
\operatorname{cd}(\mathfrak{a}, X) \geq \sup \left\{\operatorname{cd}(\mathfrak{a}, R / \mathfrak{p})-\inf X_{\mathfrak{p}} \mid \mathfrak{p} \in \operatorname{Spec} R\right\} .
$$

Moreover, equality hold if $X \in \mathcal{D}_{+}^{f}(R)$.

Proof. We may assume that $\mathrm{H}(X) \neq 0$. For $\mathfrak{p} \in \operatorname{Supp} X$, take $\ell=\inf X_{\mathfrak{p}}$ so that $\mathfrak{p} \in \operatorname{Supp}_{\ell}(X)$. Thus we have

$$
\operatorname{cd}(\mathfrak{a}, R / \mathfrak{p})-\inf X_{\mathfrak{p}} \leq \operatorname{cd}\left(\mathfrak{a}, \mathrm{H}_{\ell}(X)\right)-\ell
$$


which implies the inequality.

Now assume $X \in \mathcal{D}_{+}^{f}(R)$. Let $\ell \in \mathbb{Z}$ such that $\mathrm{H}_{\ell}(X) \neq 0$. By Theorem 1.3, $\operatorname{cd}\left(\mathfrak{a}, \mathrm{H}_{\ell}(X)\right)-\ell=\mathrm{cd}(\mathfrak{a}, R / \mathfrak{q})-\ell$, for some $\mathfrak{q} \in \operatorname{Supp~}_{\ell}(X)$, which implies cd $\left(\mathfrak{a}, \mathrm{H}_{\ell}(X)\right)-$ $\ell \leq \operatorname{cd}(\mathfrak{a}, R / \mathfrak{q})-\inf X_{\mathfrak{q}}$, the result follows.

Proposition 2.6. If $X \in \mathcal{D}_{+}^{f}(R)$ and $M$ is a finite $R$-module, then

$$
\operatorname{cd}\left(\mathfrak{a}, X \otimes_{R}^{\mathbf{L}} M\right)=\operatorname{cd}\left(\mathfrak{a}, X \otimes_{R}^{\mathbf{L}} R / \operatorname{Ann}_{R}(M)\right) \leq \operatorname{cd}(\mathfrak{a}, X) .
$$

Proof. We have $X \otimes_{R}^{\mathbf{L}} M \in \mathcal{D}_{+}^{f}(R)$ so, by Proposition 2.5,

$$
\begin{aligned}
\operatorname{cd}\left(\mathfrak{a}, X \otimes_{R}^{\mathbf{L}} M\right) & =\sup \left\{\operatorname{cd}(\mathfrak{a}, R / \mathfrak{p})-\inf \left(X \otimes_{R}^{\mathbf{L}} M\right)_{\mathfrak{p}} \mid \mathfrak{p} \in \operatorname{Spec} R\right\} \\
& =\sup \left\{\operatorname{cd}(\mathfrak{a}, R / \mathfrak{p})-\inf X_{\mathfrak{p}} \mid \mathfrak{p} \in \operatorname{Supp} X \cap \operatorname{Supp} M\right\} \\
& \leq \sup \left\{\operatorname{cd}(\mathfrak{a}, R / \mathfrak{p})-\inf X_{\mathfrak{p}} \mid \mathfrak{p} \in \operatorname{Supp} X\right\} \\
& =\operatorname{cd}(\mathfrak{a}, X) .
\end{aligned}
$$

To prove the first equality, we proceed as follows.

$$
\operatorname{cd}\left(\mathfrak{a}, X \otimes_{R}^{\mathbf{L}} M\right)=\sup \left\{\operatorname{cd}(\mathfrak{a}, R / \mathfrak{p})-\inf \left(X \otimes_{R}^{\mathbf{L}} M\right)_{\mathfrak{p}} \mid \mathfrak{p} \in \operatorname{Spec} R\right\} .
$$

By [Fo1; Lemma 16.28], we have $\inf \left(X_{\mathfrak{p}} \otimes_{R_{\mathfrak{p}}}^{\mathbf{L}} M_{\mathfrak{p}}\right)=\inf \left(X_{\mathfrak{p}} \otimes_{R_{\mathfrak{p}}}^{\mathbf{L}} R_{\mathfrak{p}} / \operatorname{Ann}_{R_{\mathfrak{p}}} M_{\mathfrak{p}}\right)$.

In $[\mathbf{A}]$, Apassov defined the weak annihilator of a complex $X \in \mathcal{D}(R)$ to be the intersection of the annihilators of all the homology modules of $X$ and denoted by $\operatorname{Ann}_{R} X$.

The following result compares cohomological dimension of $X$ with that of $R / \operatorname{Ann}_{R} X$. It is shown, in particular when $X \in \mathcal{D}_{+}^{f}(R)$, that $\operatorname{cd}(\mathfrak{a}, X)$ lies in the interval

$$
\left[\operatorname{cd}\left(\mathfrak{a}, R / \operatorname{Ann}_{R} X\right)-\sup X, \operatorname{cd}\left(\mathfrak{a}, R / \operatorname{Ann}_{R} X\right)-\inf X\right]
$$

of length $\operatorname{amp} X=\sup X-\inf X$.

Theorem 2.7. Let $X \in \mathcal{D}_{+}(R)$. Then

$$
\operatorname{cd}\left(\mathfrak{a}, R / \operatorname{Ann}_{R} X\right)-\sup X \leq \operatorname{cd}(\mathfrak{a}, X) \leq \operatorname{cd}\left(\mathfrak{a}, R / \operatorname{Ann}_{R} X\right)-\inf X .
$$

Proof. By using Proposition 2.4, we can choose $\ell \in \mathbb{Z}$ such that $\operatorname{cd}(\mathfrak{a}, X)=\operatorname{cd}\left(\mathfrak{a}, \mathrm{H}_{\ell}(X)\right)-$ $\ell$. By Lemma 2.1, $\mathrm{cd}\left(\mathfrak{a}, \mathrm{H}_{\ell}(X)\right) \leq \mathrm{cd}\left(\mathfrak{a}, R / \operatorname{Ann}_{R} X\right)$. As $\ell \geq \inf X$, the right hand side inequality follows. 
By Theorem 1.3, cd $\left(\mathfrak{a}, R / \operatorname{Ann}_{R} X\right)=\operatorname{cd}(\mathfrak{a}, R / \mathfrak{p})$ for some prime ideal $\mathfrak{p} \supseteq \operatorname{Ann}_{R} X$. Hence

$$
\begin{aligned}
\operatorname{cd}\left(\mathfrak{a}, R / \operatorname{Ann}_{R} X\right) & \leq \operatorname{cd}(\mathfrak{a}, R / \mathfrak{p})-\inf X_{\mathfrak{p}}+\sup X \\
& \leq \operatorname{cd}(\mathfrak{a}, X)+\sup X .
\end{aligned}
$$

The last inequality follows from Proposition 2.5, which implies the assertion.

The Theorem 2.9 expresses the cohomological dimension of $X \otimes_{R}^{\mathrm{L}} Y$ with the cohomological dimensions of the tensor product of the homology modules of $X$ and $Y$. But first we bring the following auxiliary result.

Proposition 2.8. If $M$ and $N$ are finite $R$-modules, then

$$
\operatorname{cd}\left(\mathfrak{a}, M \otimes_{R}^{\mathbf{L}} N\right)=\operatorname{cd}\left(\mathfrak{a}, M \otimes_{R} N\right) .
$$

Proof. We have $M \otimes_{R}^{\mathbf{L}} N \in \mathcal{D}_{+}^{f}(R)$, by [Fo1; 7.28 and 7.31], and that, by Proposition 2.5,

$$
\begin{aligned}
\operatorname{cd}\left(\mathfrak{a}, M \otimes_{R}^{\mathbf{L}} N\right) & =\sup \left\{\operatorname{cd}(\mathfrak{a}, R / \mathfrak{p})-\inf \left(M \otimes_{R}^{\mathbf{L}} N\right)_{\mathfrak{p}} \mid \mathfrak{p} \in \operatorname{Spec} R\right\} \\
& =\sup \left\{\operatorname{cd}(\mathfrak{a}, R / \mathfrak{p})-\inf \left(M_{\mathfrak{p}} \otimes_{R_{\mathfrak{p}}}^{\mathbf{L}} N_{\mathfrak{p}}\right) \mid \mathfrak{p} \in \operatorname{Spec} R\right\} \\
& =\sup \left\{\operatorname{cd}(\mathfrak{a}, R / \mathfrak{p})-\inf M_{\mathfrak{p}}-\inf N_{\mathfrak{p}} \mid \mathfrak{p} \in \operatorname{Spec} R\right\}
\end{aligned}
$$

and the assertion follows by Theorem 1.3.

Theorem 2.9. Let $X, Y \in \mathcal{D}_{+}^{f}(R)$. Then

$$
\operatorname{cd}\left(\mathfrak{a}, X \otimes_{R}^{\mathbf{L}} Y\right)=\sup \left\{\operatorname{cd}\left(\mathfrak{a}, \mathrm{H}_{t}(X) \otimes_{R} \mathrm{H}_{\ell}(Y)\right)-\ell-t \mid t, \ell \in \mathbb{Z}\right\} .
$$

Proof. We have $X \otimes_{R}^{\mathbf{L}} Y \in \mathcal{D}_{+}^{f}(R)$, c.f. [Fo1; 7.28 and 7.31]. By using [Fo1; 16.28] and Proposition 2.5, the following equalities hold.

$$
\begin{aligned}
\operatorname{cd}\left(\mathfrak{a}, X \otimes_{R}^{\mathbf{L}} Y\right) & =\sup \left\{\operatorname{cd}(\mathfrak{a}, R / \mathfrak{p})-\inf \left(X \otimes_{R}^{\mathbf{L}} Y\right)_{\mathfrak{p}} \mid \mathfrak{p} \in \operatorname{Spec} R\right\} \\
& =\sup \left\{\operatorname{cd}(\mathfrak{a}, R / \mathfrak{p})-\inf \left(X_{\mathfrak{p}} \otimes_{R_{\mathfrak{p}}}^{\mathbf{L}} Y_{\mathfrak{p}}\right) \mid \mathfrak{p} \in \operatorname{Spec} R\right\} \\
& =\sup \left\{\operatorname{cd}(\mathfrak{a}, R / \mathfrak{p})-\inf \inf _{\ell \in \mathbb{Z}}\left(\inf \left(X_{\mathfrak{p}} \otimes_{R_{\mathfrak{p}}} \mathrm{H}_{\ell}\left(Y_{\mathfrak{p}}\right)\right)+\ell \mid \mathfrak{p} \in \operatorname{Spec} R\right\}\right. \\
& =\sup \left\{\operatorname{cd}(\mathfrak{a}, R / \mathfrak{p})-\inf \left(X_{\mathfrak{p}} \otimes_{R_{\mathfrak{p}}} \mathrm{H}_{\ell}\left(Y_{\mathfrak{p}}\right)\right)-\ell \mid \mathfrak{p} \in \operatorname{Spec} R, \ell \in \mathbb{Z}\right\} \\
& =\sup \left\{\sup \left\{\operatorname{cd}(\mathfrak{a}, R / \mathfrak{p})-\inf \left(X \otimes_{R}^{\mathbf{L}} \mathrm{H}_{\ell}(Y)\right)_{\mathfrak{p}} \mid \mathfrak{p} \in \operatorname{Spec} R\right\}-\ell \mid \ell \in \mathbb{Z}\right\} \\
& =\sup \left\{\operatorname{cd}\left(\mathfrak{a}, X \otimes_{R}^{\mathbf{L}} \mathrm{H}_{\ell}(Y)\right)-\ell \mid \ell \in \mathbb{Z}\right\} .
\end{aligned}
$$


Now the assertion holds by iterating the above technique and using Proposition 2.7.

Corollary 2.10. Let $X, Y \in \mathcal{D}_{+}^{f}(R)$. Then

$$
\operatorname{cd}\left(\mathfrak{a}, X \otimes_{R}^{\mathbf{L}} Y\right) \leq \operatorname{cd}(\mathfrak{a}, X)-\inf Y
$$

Proof. It follows from Theorem 2.9 and Proposition 2.6.

\section{Cohomological dimension and the right derived section functor}

For a complex $X \in \mathcal{D}_{+}(R), \mathbf{R} \Gamma_{\mathfrak{a}}(X)$, the right derived section functor with support in $\mathrm{V}(\mathfrak{a})$ applied to the complex $X$, has been studied by several authors, e.g. [AJL], $[\mathbf{F I}]$, $[\mathbf{F o 2}],[\mathbf{F r}],[\mathbf{L}],[\mathbf{S c h}]$, and $[\mathbf{Y}]$, whom have studied the invariant $\sup \mathbf{R} \Gamma_{\mathfrak{a}}(X)$. In this section we are interested $\operatorname{in} \inf \mathbf{R} \Gamma_{\mathfrak{a}}(X)$.

The following result is a new form of [Fo2; Proposition 2.5] and the proof is similar, which H.-B. Foxby clarified to us in a private discussion.

Lemma 3.1. Let $X, Y \in \mathcal{D}_{+}(R)$. Then

$$
\inf \left(X \otimes_{R}^{\mathbf{L}} Y\right) \geq \inf \left\{\inf \left(X \otimes_{R}^{\mathbf{L}} \mathrm{H}_{\ell}(Y)\right)+\ell \mid \ell \in \mathbb{Z}\right\}
$$

Moreover, the equality holds if $X \in \mathcal{D}_{+}^{f}(R)$.

Now we are ready to prove the main result of this section.

Theorem 3.2. If $X \in \mathcal{D}_{+}(R)$, then $-\inf \mathbf{R} \Gamma_{\mathfrak{a}}(X) \leq \operatorname{cd}(\mathfrak{a}, X)$. Moreover, the equality holds if $X \in \mathcal{D}_{+}^{f}(R)$.

Proof. By [Sch; Proposition 3.2], there is a functorial isomorphism

$$
\mathbf{R} \Gamma_{\mathfrak{a}}(X) \cong \mathbf{R} \Gamma_{\mathfrak{a}}(R) \otimes_{R}^{\mathbf{L}} X .
$$

Hence we have the following. 


$$
\begin{aligned}
-\inf \mathbf{R} \Gamma_{\mathfrak{a}}(X) & =-\inf \left(\mathbf{R} \Gamma_{\mathfrak{a}}(R) \otimes_{R}^{\mathbf{L}} X\right) \\
& \leq-\inf \left\{\inf \left(\mathbf{R} \Gamma_{\mathfrak{a}}(R) \otimes_{R}^{\mathbf{L}} \mathrm{H}_{\ell}(X)\right)+\ell \mid \ell \in \mathbb{Z}\right\} \\
& =-\inf \left\{-\operatorname{cd}\left(\mathfrak{a}, \mathrm{H}_{\ell}(X)\right)+\ell \mid \ell \in \mathbb{Z}\right\} \\
& =\sup \left\{\operatorname{cd}\left(\mathfrak{a}, \mathrm{H}_{\ell}(X)\right)-\ell \mid \ell \in \mathbb{Z}\right\} \\
& =\operatorname{cd}(\mathfrak{a}, X) .
\end{aligned}
$$

The inequality follows by Lemma 3.1 and the second equality by [Sch; Proposition 3.2]. Now assume $X \in \mathcal{D}_{+}^{f}(R)$. Then the inequality becomes equality by Lemma 3.1.

To present the final result we remind the notion of $\mathfrak{a}-\operatorname{depth} X$, which is denoted by $\operatorname{depth}(\mathfrak{a}, X)$, and defined by

$$
\operatorname{depth}(\mathfrak{a}, X)=-\sup \mathbf{R} \operatorname{Hom}_{R}(R / \mathfrak{a}, X) .
$$

In [FI; Theorem 2.1], Foxby and Iyengar show that $\operatorname{depth}(\mathfrak{a}, X)=-\sup \mathbf{R} \Gamma_{\mathfrak{a}}(X)$.

Theorem 3.3. Let $R$ be local, $X \in \mathcal{D}_{+}(R)$ and that depth $(\mathfrak{a}, X)<\infty$. Then

$$
\operatorname{depth}(\mathfrak{a}, X) \leq \operatorname{cd}(\mathfrak{a}, X) .
$$

Proof. One has

$$
\begin{aligned}
\operatorname{depth}(\mathfrak{a}, X) & =-\sup \mathbf{R} \Gamma_{\mathfrak{a}}(X) \\
& \leq-\inf \mathbf{R} \Gamma_{\mathfrak{a}}(X) \\
& \leq \operatorname{cd}(\mathfrak{a}, X) .
\end{aligned}
$$

Now the assertion holds.

We end this paper with two questions.

1. For any $X \in \mathcal{D}(R)$ and $\mathfrak{p} \in \operatorname{Supp} X$ we have the inequality of dimensions $\operatorname{dim} X_{\mathfrak{p}}+$ $\operatorname{dim} R / \mathfrak{p} \leq \operatorname{dim} X$, cf. [Fo1; 16.16]. Is there any similar inequality for cohomological dimensions?

2. If $X \in \mathcal{D}_{+}^{f}(R)$ is not homologically trivial and $Y \in \mathcal{D}_{b}^{f}(R)$ then by the Intersection Theorem we have the following inequality $\operatorname{dim} Y \leq \operatorname{dim}\left(X \otimes_{R}^{\mathbf{L}} Y\right)+\operatorname{proj} \cdot \operatorname{dim} X$. Is there any similar inequality for the cohomological dimensions, such as

$$
\operatorname{cd}(\mathfrak{a}, Y) \leq \operatorname{cd}\left(\mathfrak{a}, X \otimes_{R}^{\mathbf{L}} Y\right)+\text { proj.dim } X ?
$$


Acknowledgments. The authors would like to thank H.-B. Foxby, University of Copenhagen, for his invaluable help, specially for posting the latest version of the reference [Fo1]. The research of the first author was partially supported by a grant from IPM, and that of the second author was partially supported by a grant from University of Tehran.

\section{References}

[A] D. Apassov, Annihilating complexes of modules, Math. Scand. 84 (1999), $11-22$

[AJL] L. Alonso Tarrío, A. Jeremías López, J. Lipman, Local homology and cohomology on schemes, Ann. Sci. École Norm. Sup. (4) 30 (1997), 1-39.

[DNT] K. Divaani-Aazar, R. Naghipour, M. Tousi, Cohomological dimension of certain algebraic varieties, Proc. Amer. Math. Soc. 130 (2002), no. 12, $3537-3544$.

[F] G. Falting, Über lokale Kohomologiegruppen hoher Ordnung, J. Reine Angew. Math. 313 (1980), 43-51.

[G] A. Grothendieck, Cohomologie locale des faisceaux cohérents et théorèmes de Lefschetz locaux et globaux (SGA 2), Augmenté dun exposé par Michèle Raynaud. Séminaire de Géométrie Algébrique du Bois-Marie, 1962. Advanced Studies in Pure Mathematics, Vol. 2. North-Holland Publishing Co., Amsterdam; Masson \& Cie, Éditeur, Paris, 1968.

[Ha1] R. Hartshorne, Residues and duality, Lecture Notes in Math., 20, Springer Verlag, 1971.

[Ha2] R. Hartshorne, Cohomological dimension of algebraic varieties, Ann. of Math. (2) 88 (1968), 403-450.

[Hu] C. Huneke, Problems on local cohomology, Free resolutions in commutative algebra and algebraic geometry (Sundance, UT, 1990), 93-108, Res. Notes Math., 2, Jones and Bartlett, Boston, MA, 1992. 
[HL] C. Huneke, G. Lyubeznik, On the vanishing of local cohomology modules Invent. Math. 102 (1990), no. 1, 73-93.

[Fo1] H.-B. Foxby, Hyperhomological algebra and commutative algebra, Notes in preparation.

[Fo2] H.-B. Foxby, Bounded complexes of flat modules, J. Pure Appl. Algebra 15 (1979), 149-172.

[FI] H.-B. Foxby, S. Iyengar, Depth and amplitude for unbounded complexes, to appear in Contemporary Math.

[Fr] A. Frankild, Vanishing of local homology, to appear in Math. Z.

[GM] J. P. C. Greenlees, J. P. May, Derived functors of I-adic completion and local homology, J. Algebra 149 (1992), 438-453.

[I] S. Iyengar, Depth for complexes, and intersection theorems, Math. Z. 230 (1999), 545-567.

[L] J. Lipman, Lectures on local cohomology and duality. Local cohomology and its applications (Guanajuato, 1999), 39-89, Lecture Notes in Pure and Appl. Math., 226, Dekker, New York, 2002.

[Sch] P. Schenzel, Proregular sequences, local cohomology, and completions, preprint.

[Y] S. Yassemi, Generalized section functors, J. Pure Appl. Algebra 95 (1994), 103-119.

E-mail addresses:

dibaeimt@ipm.ir

yassemi@ipm.ir 